\title{
First Principles Evolutionary Search for New Electrides along the Dimensionality of Anionic Electrons
}

\author{
Tomofumi TADA ${ }^{\mathrm{a},{ }^{*}}$, Junjie WANG ${ }^{\mathrm{a}, \mathrm{b}}$, and Hideo HOSONO ${ }^{\mathrm{a}, \mathrm{c}}$ \\ ${ }^{a}$ Materials Research Center for Element Strategy, Tokyo Inst. of Technology, \\ 4259-SE3 Nagatsuta-cho, Midori-ku, Yokohama, Kanagawa, 226-8501, Japan \\ ${ }^{\mathrm{b}}$ International Center for Materials Nanoarchitectonics (MANA), National Institute for Materials Science, \\ Ibaraki 305-0044, Japan \\ ${ }^{c}$ Laboratory for Materials and Structures, Institute of Innovative Research, Tokyo Inst. of Technology, \\ 4259 Nagatsuta-cho, Midori-ku, Yokohama, Kanagawa, 226-8501, Japan \\ *E-mail: tada.t.ae@m.titech.ac.jp \\ January 30, 2018
}

(Received: December 12, 2017; Accepted for publication: January 9, 2018; Online publication: February 20, 2018)

We propose a unique strategy based on the dimensionality of anionic electrons for new inorganic electride search. We adopted strontium phosphide as the initiator for the evolutionary search, and our evolutionary calculations combined with experimental synthesis found the $\mathrm{Sr}_{5} \mathrm{P}_{3}$ is a new one-dimensional electride.

Keywords: Electride, Evolutionary search, First principles.

\section{Introduction}

Electride is a unique material which serves a bare electron as anion, and we thereby expect chemical activity of electride, which was in fact already confirmed in inorganic electrides $\mathrm{C} 12 \mathrm{~A} 7$ [1] and $\mathrm{Ca}_{2} \mathrm{~N}$ [2] for ammonia synthesis [3]. What's the next electride is thus one of the most exciting issues in Inorganic chemistry. Recently, we reported a new electride strontium phosphide which was firstly predicted with first principles evolutionary search and succeeded in experimental synthesis [4]. The evolutionary search is quite a powerful tool in theoretical exploration for unknown compounds. Once we provide the elements constituting target compounds, the formula and corresponding stable structures are automatically identified with the first principles evolutionary search. The elements we selected in the previous study were $\mathrm{Sr}$ and P, i.e., strontium phosphide, and if this selection were inappropriate, the evolutionary search would fail. That is, the preconditioning by human imagination before computational screening by artificial intelligence such as an evolutionary search is quite an important step for unknown compounds search. In this paper, we explain how we focused on strontium phosphide as a candidate for new electride, which was done along the dimensionality of anionic electrons.

\section{Method}

Let us firstly categorize inorganic electride in terms of dimensions: zero-dimensional (0D), one-D (1D), two-D (2D), and three-D (3D). Figure 1 shows schematics and sample compounds in each category, although the sample for $3 \mathrm{D}$ electride is a hypothetical one [6]. Here we introduce a crude assumption that the stability of an inorganic electride could be related to the interactions between anionic electrons [7], although there are other types of ionic interactions in the system. When we accept the assumption, the anionic electron in $0 \mathrm{D}$ electrides is stable because it is almost isolated from other anionic electrons. On the other hand, for a 3D electride, anionic electron is surrounded by many anionic electrons which would destabilize the 3D electride compared with $0 \mathrm{D}$ 
(a)

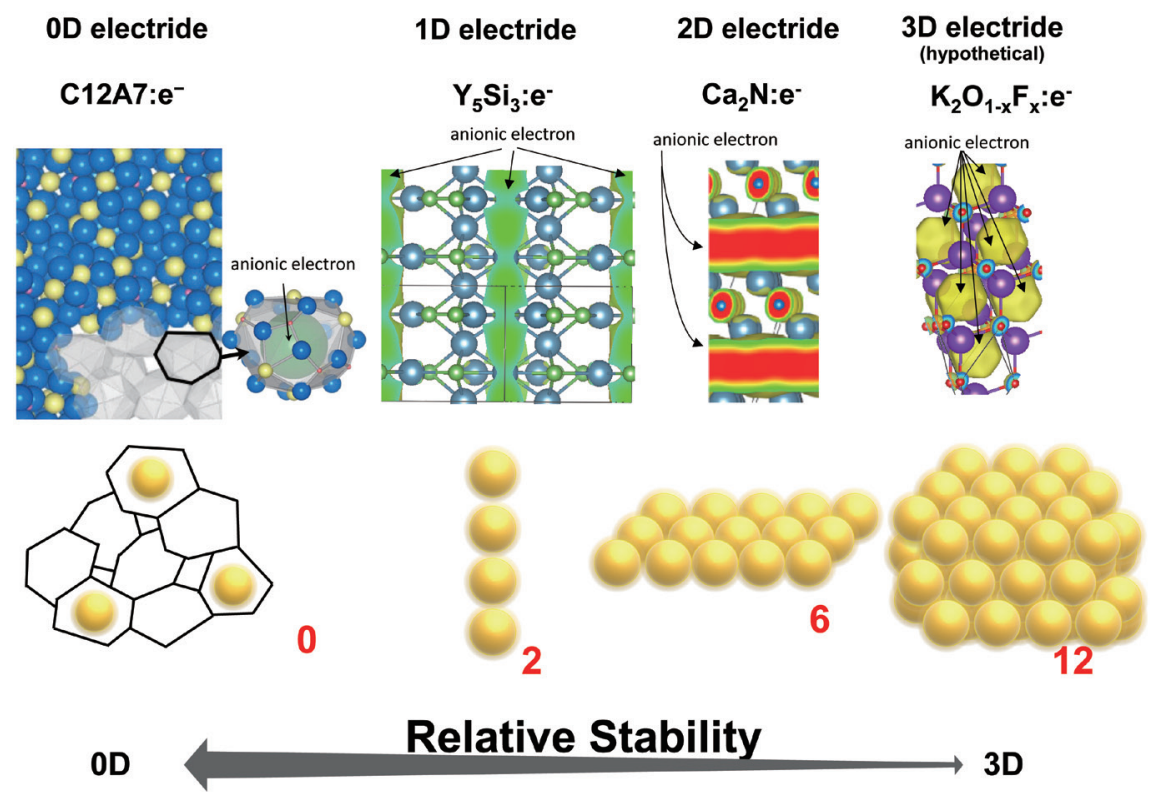

(b)

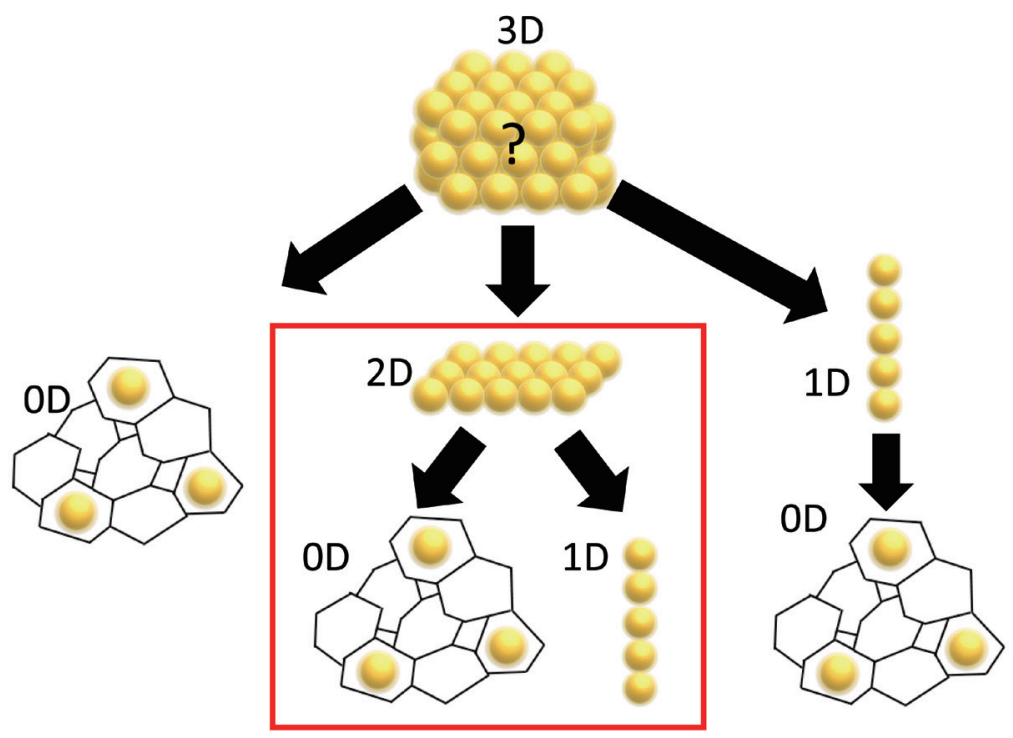

Figure 1. Schematic of the searching strategy based on the dimensionality of anionic electrons. (a) Inorganic electrides with 0D (reprinted with permission from Ref [5]. Copyright 2013 Nature Publishing Group), 1D, 2D, and 3D network of anionic electrons. The numbers with red indicate the number of neighboring anionic electrons. (b) The concept of the stability of anionic electrons by lowering the dimensionality of the network.

electride. In this sense, the stability of anionic electrons in inorganic electrides can be written as $0 \mathrm{D}>1 \mathrm{D}>2 \mathrm{D}>3 \mathrm{D}$.

Let us next consider what strategy is efficient to find new electrides. Since the stability of inorganic electrides would follow the order of $0 D>1 D>2 D>3 D$, when we identify the candidate for $3 \mathrm{D}$ electride as $\mathrm{A}_{n} \mathrm{~B}_{m}$, the compounds $\mathrm{A}_{n} \mathrm{~B}_{m}$ can also have a chance to appear as $2 \mathrm{D}, 1 \mathrm{D}$, and $0 \mathrm{D}$ electrides even when the synthesis of $3 \mathrm{D}$ electride $\mathrm{A}_{n} \mathrm{~B}_{m}$ is failed. In the same 
Table 1. Relative stability of 2D electrides. $E_{\text {diff }}$ indicates the relative stability of $2 \mathrm{D}$ electride compared with reference compounds; negative/positive number means stable/unstable.

\begin{tabular}{ccc}
\hline compounds & $\mathbf{E}_{\text {diff }}(\mathbf{e V} /$ atom $)$ & References \\
\hline $\mathrm{Ca}_{2} \mathrm{~N}$ & -0.051 & $\mathrm{Ca}_{3} \mathrm{~N}_{2}, \mathrm{Ca}$ \\
$\mathrm{Sr}_{2} \mathrm{~N}$ & -0.167 & $\mathrm{SrN}, \mathrm{Sr}$ \\
$\mathrm{Ba}_{2} \mathrm{~N}$ & -0.042 & $\mathrm{Ba}_{3} \mathrm{~N}, \mathrm{BaN}$ \\
$\mathrm{Ca}_{2} \mathrm{P}$ & -0.083 & $\mathrm{CaP}, \mathrm{Ca}$ \\
$\mathrm{Sr}_{2} \mathrm{P}$ & +0.053 & $\mathrm{Sr}_{3} \mathrm{P}_{2}, \mathrm{Sr}$ \\
$\mathrm{Ba}_{2} \mathrm{P}$ & -0.003 & $\mathrm{Ba}_{3} \mathrm{P}_{2}, \mathrm{Ba}$ \\
$\mathrm{Ca}_{2} \mathrm{As}$ & +0.185 & $\mathrm{Ca}_{5} \mathrm{As}_{3}, \mathrm{Ca}$ \\
$\mathrm{Sr}_{2} \mathrm{As}$ & +0.102 & $\mathrm{Sr}_{5} \mathrm{As}_{3}, \mathrm{Sr}$ \\
$\mathrm{Ba}_{2} \mathrm{As}$ & +0.032 & $\mathrm{Ba}_{20} \mathrm{P}_{13}, \mathrm{Ba}$ \\
$\mathrm{Ca}_{2} \mathrm{Sb}$ & +0.130 & $\mathrm{Ca}_{5} \mathrm{Sb}_{3}, \mathrm{Ca}$ \\
$\mathrm{Sr}_{2} \mathrm{Sb}$ & +0.210 & $\mathrm{Sr}_{5} \mathrm{Sb}_{3}, \mathrm{Sr}$ \\
$\mathrm{Ba}_{2} \mathrm{Sb}$ & +0.134 & $\mathrm{Ba}_{5} \mathrm{Sb}_{3}, \mathrm{Ba}$ \\
$\mathrm{Ca}_{2} \mathrm{Bi}$ & +0.163 & $\mathrm{Ca}_{5} \mathrm{Bi}_{3}, \mathrm{Ca}$ \\
$\mathrm{Sr}_{2} \mathrm{Bi}$ & +0.137 & $\mathrm{Sr}_{5} \mathrm{Bi}_{3}, \mathrm{Sr}$ \\
$\mathrm{Ba}_{2} \mathrm{Bi}$ & +0.158 & $\mathrm{Ba}_{5} \mathrm{Bi}_{3}, \mathrm{Ba}$ \\
\hline
\end{tabular}

sense, the candidates for $2 \mathrm{D}$ electride $\mathrm{X}_{n} \mathrm{Y}_{m}$ would be expected to appear as $0 \mathrm{D}$ or $1 \mathrm{D}$ electride. This is our dimensional strategy for new inorganic electrides. Since we already know $\mathrm{Ca}_{2} \mathrm{~N}$ [2] and $\mathrm{Y}_{2} \mathrm{C}[8]$ are $2 \mathrm{D}$ electrides, the dimensional strategy starting from 2D electride is a practical one.

To determine the elements for new electride search, we investigated the relative stability of hypothetical $2 \mathrm{D}$ electride of alkaline earth $\left(A_{e}\right)$ pnictide $(P)$ as listed in Table 1; only $\mathrm{Ca}_{2} \mathrm{~N}$ is a realistic $2 \mathrm{D}$ electride. The space group of the compounds is fixed to be R-3m which is identical to that of $\mathrm{Ca}_{2} \mathrm{~N}$. The electronic structure calculations and structural relaxations were carried out using density functional theory (DFT) with PAW method implemented in VASP [9]. Based on the formal charges of ions, we adopted the formula of $\left(A_{e}\right)_{2} P$ to find anionic electrons in the crystals. For example, $\mathrm{Ca}_{2} \mathrm{~N}$ is composed of $\mathrm{Ca}^{2+}$ and $\mathrm{N}^{3-}$, and thus one electron is expected to appear as a bare electron to hold the charge neutrality, that is, $\mathrm{Ca}_{2} \mathrm{~N}: \mathrm{e}^{-}$. In the same manner, the hypothetical formula of alkaline earth pnictides was adopted in the investigation of the relative stability. In Table 1, the most stable stoichiometric binary compounds were selected as References according to Materials Project database [10]; only when a 2D electride already appears in the database as the most stable one, we used second stable binary compounds as References.

\section{Results and discussion}

Calculated relative energies with respect to reference compounds listed in Table 1 show that 2D electrides of nitrides are stable, which corresponds to the fact that $\mathrm{Ca}_{2} \mathrm{~N}$ is an experimentally validated 2D electride. On the other hand, the arsenide, antimonide, and bithmus compounds are clearly unstable. The phosphides showing a slight stability could be candidates for new electride search, and we selected strontium phosphide, $\mathrm{Sr}_{n} \mathrm{P}_{m}$.

Now that we have fixed the target compounds $\operatorname{Sr}_{n} \mathrm{P}_{m}$ for new electride, the next step is the first principles evolutionary search and we adopted USPEX code [11] coupled with VASP. At the beginning of the process, a first generation of structures is produced using a random-number generator, and structural relaxation is carried out with VASP for each structure to find stable crystal structures. After the first generation process, USPEX creates the second generation by keeping the most stable structure and by combining relatively stable structures through variation operations based on the evolutionary algorithm (such as heredity, permutation, mutation, soft mutation...). In this manner, the generation is updated based on the previous generation, and the update is finished when the most stable structure is unchanged over the course of 40 generations.

Since much more details of this process are written in our paper [4], we summarize the findings in the evolutionary study on $\mathrm{Sr}_{n} \mathrm{P}_{m}$ : 1) 2D electride $\mathrm{Sr}_{2} \mathrm{P}$ with R-3m structure is a meta stable structure from the ambient pressure to $15 \mathrm{GPa}, 2) 1 \mathrm{D}$ electride $\mathrm{Sr}_{5} \mathrm{P}_{3}$ with $\mathrm{C} 2 / \mathrm{m}$ structure is the most stable structure at the ambient pressure, and the structure is transformed to a higher symmetry P63/mcm above $10 \mathrm{GPa}$ keeping the onedimensional network of anionic electrons (i.e., 1D electride), 3) Experimentally synthesized strontium phosphide is $\mathrm{Sr}_{5} \mathrm{P}_{3}$ with $\mathrm{C} 2 / \mathrm{m}$ structure, 4) A discrepancy on the band gap between the experimental and DFT results is found; insulator by experiment but metal by calculation (DFT), and the discrepancy is rationalized as the evidence of Motto insulator of $\mathrm{Sr}_{5} \mathrm{P}_{3}$ 1D electride, and 5) 0D electride $\mathrm{Sr}_{8} \mathrm{P}_{5}$ with I-4 structure, a competing phase against the $1 \mathrm{D}$ electride $\mathrm{Sr}_{5} \mathrm{P}_{3}$, can appear at the ambient pressure. These results indicate the powerfulness of the evolutionary search along the dimensional point of anionic electrons, combined with experimental synthesis. 


\section{Summary}

A unique strategy based on the dimensionality of anionic electrons was adopted in an evolutionary search for new electrides. According to the strategy, we adopted strontium phosphide as the initiator for the evolutionary search, and we found 1D electride $\mathrm{Sr}_{5} \mathrm{P}_{3}$ and $0 \mathrm{D}$ electride $\mathrm{Sr}_{8} \mathrm{P}_{5}$. The dimensional strategy will be useful for searching for other unknown inorganic electrides.

This work was supported by the Ministry of Education, Culture, Sports, Science and Technology (MEXT) through the Element Strategy Initiative to Form Core Research Center. This work was supported in part by the ACCEL program sponsored by the Japan Science and Technology Agency. TT, and HH were also supported by the Japan Society for the Promotion of Science (JSPS) through a Grant-in-Aid for Scientific Research (S) No. 17H06153.

\section{References}

[1] S. Matsuishi, Y. Toda, M. Miyakawa, K. Hayashi, T. Kamiya, M. Hirano, I. Tanaka, H. Hosono, Science, 301, 626 (2003). DOI:10.1126/science.1083842

[2] K. Lee, S. W. Kim, Y. Toda, S. Matsuishi, H. Hosono, Nature, 494, 336 (2013). DOI:10.1038/nature 11812

[3] M. Kitano, Y. Inoue, Y. Yamazaki, F. Hayashi, S. Kanbara, S. Matsuishi, T. Yokoyama, S. W. Kim, M. Hara, H. Hosono, Nat. Chem., 4, 934 (2012). DOI:10.1038/ nchem. 1476
[4] J. Wang, K. Hanzawa, H. Hiramatsu, J. Kim, N. Umezawa, K. Iwanaka, T. Tada, H. Hosono, J. Am. Chem. Soc., 139, 15668 (2017). DOI:10.1021/jacs.7b06279

[5] Y. Toda, H. Hirayama, N. Kuganathan, A. Torrisi, P. V. Sushko, and H. Hosono, Nat. Commun., 4, 2378/1-8 (2013).

[6] T. Tada, S. Takemoto, S. Matsuishi, H. Hosono, Inorg. Chem., 53, 10347 (2014). DOI:10.1021/ic501362b

[7] There is no clear validation for the assumption that the stability of inorganic electride could be represented in terms of the interactions between anionic electrons, and it's rather a concept which has to be validated in experimental and computational study. Although we don't have such a validation, we can roughly consider as follows. The spatial distribution of anionic electron in inorganic electride is determined by the positions of cations and anions, that is, the shape of anionic electron is modulated by the cations and anions easily. Bearing in mind that anionic electron might be captured by orbitals of cations in some systems, once we find anionic electrons in a system, we can recognize that the anionic electrons in the system show a slightly larger stability at the interstitial position than captured by orbitals of cations. Since the competition between the two situation is so naive, finding electride is not so easy. In other words, the local situation of anionic electrons embedded in ionic crystals could be similar, regardless of the local crystal structures. If this is true, the remaining interactions related to the stability are the interactions between anionic electrons.

[8] X. Zhang, Z. Xiao, H. Lei, Y. Toda, S. Matsuishi, T. Kamiya, S. Ueda, H. Hosono, Chem. Mater., 26, 6638 (2014). DOI:10.1021/cm503512h

[9] G. Kresse, J. Furthmüller, Comput. Mater. Sci., 6, 15 (1996). DOI:10.1016/0927-0256(96)00008-0

[10] https://materialsproject.org/

[11] A. R. Oganov, C. W. Glass, J. Chem. Phys., 124, 244704 (2006). DOI:10.1063/1.2210932 